\title{
Reflecting on the past and planning for the future
}

$\mathrm{T}$ his new volume provides me with the opportunity to look back on my first 6 hectic months at the helm of our great journal, to highlight our accomplishments and to give you a glimpse into forthcoming changes.

Our accomplishments include a major overhaul of editorials. As promised, we have assembled an interdisciplinary editorial-writing team that provides our journal with a strong independent voice. Our focus has been on public accountability, transparency and governance in the areas of health services and policy, and our discourse has clear messages with unambiguous recommendations. This may, at times, have shaken some readers, but hopefully it has also engaged them and helped to stimulate informed public debate. Do not expect our voice to change. Instead, look forward to editorials on more clinical topics in the coming months.

Other important accomplishments and changes have not been as visible as the changes made to the editorials. Considerable time was spent developing a growth plan for the journal and in rebuilding a world-class team. More specifically, we have identified new editorial board members and associate editors, reconfigured the Journal Oversight Committee and hired new staff in virtually all departments. These may not seem like earth-shattering changes, but to be a worldclass journal you need a world-class team.

These staffing changes have resulted in a substantial improvement in the peer review process, including the tracking of manuscripts and the timeliness of peer reviews. Our team of editors is also striving to improve its reviews and its remits to authors. Our goal is to improve our communication with authors and to ensure that all of our contributors are treated fairly. Hopefully, these interactions ultimately will result in a substantial improvement of every manuscript, whether it is accepted for publication in $C M A J$ or not.

Readers should also look forward to more systematic reviews in our Research section. Systematic reviews, when properly conducted and reported, provide the best evidence for therapeutic decisions. With the Canadian Cochrane Collaboration, we hope to provide researchers who undertake Cochrane reviews with another major medical journal outlet to ensure the widest possible exposure of their findings. We plan to form similar partnerships with other major Canadian agencies. The length of research articles will also be in+ creased to allow authors to elaborate on their findings.

In the Practice section, we will increase the number of narrative reviews, provide readers with literature scanning and appraisal, and enhance continuing medical education. We will also provide a forum for recognized experts who give grand rounds at different institutions to publish patientfocused clinical reviews. We hope to host podcasts and Web- based videos of the best presentations. Drug alerts and innovations in medicine will also grace the pages of CMAJ. This summer and fall you will see a few series, with more to come. Finally, expect to see both print and electronic versions of several supplements of clinical practice guidelines.

In an effort to provide a forum for physicians on the front lines of medicine, we are introducing an occasional column in the News section called "Dispatches" (see page 26). Health care professionals working in difficult circumstances or with new interventions are encouraged to submit a 350-word account of the challenges they face. We also plan to publish more international news by fostering contributions from far-flung places. Forthcoming issues will include pieces on Canadian physicians at work in Afghanistan and Iraq, as well as a report on the state of health care in France.

To improve the clarity of voices in $C M A J$, we will now be highlighting author degrees and affiliations. We also plan to extend our reach by providing abstracts in French, both in print and online, for readers who prefer la langue de Molière. There also will be additional French content on CMAJ.ca, in the form of commentaries, Cochrane review abstracts and elements of public health.

The past volume bids a fond adieu to our back-page columnist, Dr. Ursus, who managed both to entertain and to impart wisdom by allowing us into his life. But we are excited to introduce "Salon," an exchange of lateral thinking about health that aspires to feed the culture of curiosity and nurture the intellect. $C M A$ 's salon will explore nascent ideas and hypotheses that are fuelled by fact and driven by passion.

Despite these accomplishments and changes, there remain many challenges in growing CMAJ. As in my first editorial (CMAJ 2007;176:9), I again ask for your support. Prospective authors, consider $C M A J$ as you complete your research. Remember that $C M A J$ is heavily cited and reaches deep into the community, as its studies are typically featured in the national media every 2 weeks. CMAJ.ca has over 20 million hits per year from around the world and, most importantly, $C M A J$ is one of the world's only truly open-access major medical journals.

To help maintain that status, I ask our faithful readers to complete our forthcoming readership survey, so that we can better understand your medical information needs and so that you can help make our great journal's future look even more promising.

\section{Paul C. Hébert MD MHSc \\ Editor-in-Chief, CMAJ \\ With the Editorial-Writing Team (Matthew Stanbrook, Barbara Sibbald, Ken Flegel, Noni MacDonald and Amir Attaran)}

